Louisiana State University

LSU Digital Commons

$1-1-2004$

\title{
Measurement and control of the frequency chirp rate of high- order harmonic pulses
}

\author{
J. Mauritsson \\ Lunds Tekniska Högskola \\ P. Johnsson \\ Lunds Tekniska Högskola \\ R. López-Martens \\ Lunds Tekniska Högskola \\ K. Varjú \\ Lunds Tekniska Högskola \\ W. Kornelis \\ ETH Zürich
}

See next page for additional authors

Follow this and additional works at: https://digitalcommons.Isu.edu/physics_astronomy_pubs

\section{Recommended Citation}

Mauritsson, J., Johnsson, P., López-Martens, R., Varjú, K., Kornelis, W., Biegert, J., Keller, U., Gaarde, M., Schafer, K., \& L'Huillier, A. (2004). Measurement and control of the frequency chirp rate of high-order harmonic pulses. Physical Review A - Atomic, Molecular, and Optical Physics, 70 (2) https://doi.org/ 10.1103/PhysRevA.70.021801

This Article is brought to you for free and open access by the Department of Physics \& Astronomy at LSU Digital Commons. It has been accepted for inclusion in Faculty Publications by an authorized administrator of LSU Digital Commons. For more information, please contact ir@lsu.edu. 


\section{Authors}

J. Mauritsson, P. Johnsson, R. López-Martens, K. Varjú, W. Kornelis, J. Biegert, U. Keller, M. B. Gaarde, K. J. Schafer, and A. L'Huillier

This article is available at LSU Digital Commons: https://digitalcommons.Isu.edu/physics_astronomy_pubs/2169 


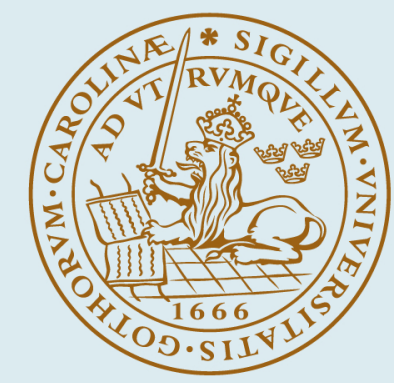

\section{LUND UNIVERSITY}

\section{Measurement and control of the frequency chirp rate of high-order harmonic pulses}

Mauritsson, Johan; Johnsson, Per; Lopez, Rodrigo; Varju, Katalin; Kornelis, W; Biegert, J; Keller, U; Gaarde, MB; Schafer, KJ; L'Huillier, Anne

Published in:

Physical Review A (Atomic, Molecular and Optical Physics)

DOI:

10.1103/PhysRevA.70.021801

2004

Link to publication

Citation for published version (APA):

Mauritsson, J., Johnsson, P., Lopez, R., Varju, K., Kornelis, W., Biegert, J., Keller, U., Gaarde, MB., Schafer, KJ., \& L'Huillier, A. (2004). Measurement and control of the frequency chirp rate of high-order harmonic pulses. Physical Review A (Atomic, Molecular and Optical Physics), 70(2), [021801]. https://doi.org/10.1103/PhysRevA.70.021801

Total number of authors:

10

\section{General rights}

Unless other specific re-use rights are stated the following general rights apply:

Copyright and moral rights for the publications made accessible in the public portal are retained by the authors and/or other copyright owners and it is a condition of accessing publications that users recognise and abide by the legal requirements associated with these rights.

- Users may download and print one copy of any publication from the public portal for the purpose of private study or research.

- You may not further distribute the material or use it for any profit-making activity or commercial gain

- You may freely distribute the URL identifying the publication in the public portal

Read more about Creative commons licenses: https://creativecommons.org/licenses/

Take down policy

If you believe that this document breaches copyright please contact us providing details, and we will remove

access to the work immediately and investigate your claim. 


\title{
Measurement and control of the frequency chirp rate of high-order harmonic pulses
}

\author{
J. Mauritsson, ${ }^{1}$ P. Johnsson, ${ }^{1}$ R. López-Martens, ${ }^{1}$ K. Varjú, ${ }^{1}$ W. Kornelis, ${ }^{2}$ J. Biegert, ${ }^{2}$ U. Keller, ${ }^{2}$ M. B. Gaarde,${ }^{3}$ \\ K. J. Schafer, ${ }^{3}$ and A. L'Huillier ${ }^{1}$ \\ ${ }^{1}$ Department of Physics, Lund Institute of Technology, P. O. Box 118, SE-221 00 Lund, Sweden \\ ${ }^{2}$ Physics Department, Swiss Federal Institute of Technology (ETH Zürich), CH-8093 Zürich, Switzerland \\ ${ }^{3}$ Department of Physics and Astronomy, Louisiana State University, Baton Rouge, Louisiana 70803-4001, USA
}

(Received 28 January 2004; published 31 August 2004)

\begin{abstract}
We measure the chirp rate of harmonics 13 to 23 in argon by cross correlation with a 12 femtosecond probe pulse. Under low ionization conditions, we directly measure the negative chirp due to the atomic dipole phase, and show that an additional chirp on the pump pulse is transferred to the $q$ th harmonic as $q$ times the fundamental chirp. Our results are in accord with simulations using the experimentally measured $815 \mathrm{~nm}$ pump and probe pulses. The ability to measure and manipulate the harmonic chirp rate is essential for the characterization and optimization of attosecond pulse trains.
\end{abstract}

DOI: 10.1103/PhysRevA.70.021801

PACS number(s): 42.65.Ky, 32.80.Rm

High harmonic generation provides a versatile laboratory source of ultrafast extreme ultraviolet (XUV) radiation [1]. Single harmonics can be used for a variety of applications, e.g., in time-resolved studies of molecular dynamics $[2,3]$. The coherent superposition of several harmonics can produce attosecond bursts [4-6] for the study of intra-atomic electron dynamics $[7,8]$. Recently, methods have been developed to measure the time-dependent frequency of individual harmonics [9-11], which is a direct measure of their coherence properties. An important application of these measurements is in the characterization of the relative coherence between successive harmonics, since this determines the temporal properties of their superposition. Indeed, control of the relative coherence between several harmonics allows both the duration and spacing of the attosecond bursts to be tailored for specific applications.

The time-dependent frequency of the emitted radiation is strongly related to the dynamics of the generation process. Each harmonic contains contributions from several spacetime quantum orbits [12-14] with different phase behaviors, which can be written as $\phi_{q}^{j}(t)=-\alpha_{q}^{j} I(\vec{r}, t)$, with $I(\vec{r}, t)$ denoting the laser intensity and $\alpha_{q}^{j}$ a coefficient depending on the process order $(q)$ and on the quantum path $(j)$. Macroscopic phase-matching conditions can favor one of these quantum paths which, in combination with ionization effects, will then determine the harmonic coherence properties. A measurement of the individual harmonic chirps can therefore be used to characterize the harmonic generation process itself, when ionization is low [15], or to probe macroscopic timedependent processes such as blueshifting, depletion, and defocusing [11] when ionization is high.

In this communication we measure and manipulate the time-dependent frequency of harmonics 13 through 23 generated in argon with $815 \mathrm{~nm}$ light. The experiment has been performed in such a way that ionization effects have been minimized, and the harmonic flux has been kept approximately constant as we vary the spectral phase of the driving laser field. We find that in our experimental conditions we select predominantly harmonics generated via the shortest quantum path, and we directly measure its order-dependent phase coefficient $\alpha_{q}=\alpha_{q}^{1}$. Previously, only indirect measurements of $\alpha_{q}$ have been performed [13,14]. In addition, we show that a chirp applied to the driving laser pulse is transferred to the $q$ th harmonic as $q$ times the fundamental chirp. The comparison of our results to detailed simulations is consistent with the interpretation that this imposed chirp simply adds to the intrinsic chirp [16]. This allows us to alter the spacing between the pulses in an attosecond train, as we increase or decrease the chirp rates of the harmonics used to synthesize the train.

The time-frequency structure of the XUV pulses is characterized via cross-correlation frequency-resolved optical gating (XFROG) [17], as described in [9]. The signal used for the cross correlation is the photoelectrons resulting from the two-photon ionization of argon by the XUV pulses and an IR probe pulse (see Fig. 1). We use transform-limited $12 \mathrm{fs}$ pulses generated using self-phase modulation in an argon-filled hollow fiber, followed by compression using chirped mirrors [18], to probe the harmonics of the transform-limited or chirped $815 \mathrm{~nm}$ fundamental pulses of

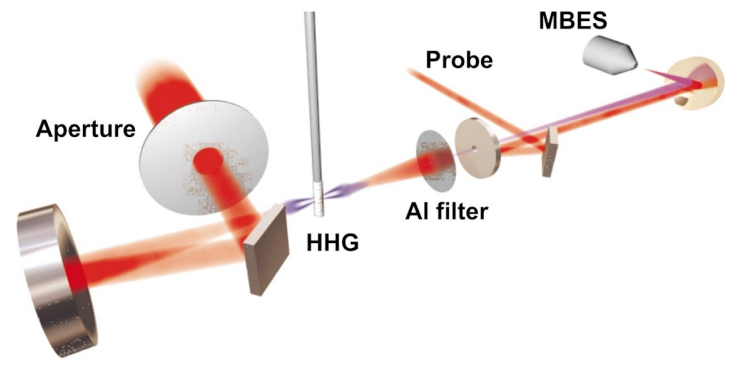

FIG. 1. (Color) Experimental setup. The harmonics are generated by focusing part of the beam into a $3 \mathrm{~mm}$, windowless cell filled with 30 mbar of argon and passed through a $200 \mathrm{~nm}$ thick aluminum foil in order to eliminate any residual IR light, and then through a $1 \mathrm{~mm}$ aperture located $35 \mathrm{~cm}$ from the generation source. The XUV light is focused by an $f=+20 \mathrm{~cm}$ normal incidence, goldcoated, spherical mirror into a MBES, filled with a $1 \times 10^{-4} \mathrm{mbar}$ background pressure of argon. The probe pulse is focused into the MBES by the same mirror as the harmonics. 


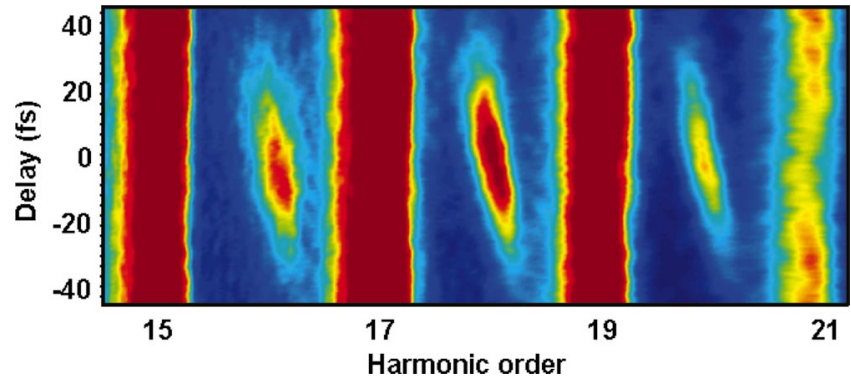

FIG. 2. (Color) Photoelectron signal obtained in Ar as a function of energy and time delay between the XUV and IR pulses.

duration between 35 and 90 fs. Both pulses are continuously monitored using two online spectral phase interferometry for direct electric-field reconstruction (SPIDER) devices [19-21]. The foci of the harmonic and probe beams are separated in the propagation direction due to the different divergence of the two beams; the harmonics are focused in the sensitive region of the magnetic bottle electron spectrometer (MBES), while the IR field is focused slightly before it. This yields an IR focus in the sensitive region of the MBES that is larger than the XUV focus, which ensures a homogeneous IR distribution over the XUV focus. The polarization vectors of the two fields are parallel and coincide with the direction of electron detection. The accuracy of our cross-correlation method depends on both the probe duration and the MBES resolution. Using a short probe pulse, as we do in this work,
TABLE I. Fundamental pulse parameters (GDD, duration, chirp rate, intensity), aperture diameter, relative harmonic flux, measured and simulated duration and chirp rate for sideband 18 .

\begin{tabular}{lccccc}
\hline \hline & $(\mathrm{a})$ & $(\mathrm{b})$ & $(\mathrm{c})$ & $(\mathrm{d})$ & $(\mathrm{e})$ \\
\hline $\mathrm{GDD}_{\text {fund }}\left(\mathrm{s}^{2}\right)$ & -1 & -0.6 & 0 & 0.3 & $0.6 \times 10^{-27}$ \\
$\tau_{\text {fund }}(\mathrm{fs})$ & 90 & 44 & 37 & 42 & 57 \\
$b_{\text {fund }}\left(\mathrm{s}^{-2}\right)$ & -0.8 & -1.1 & 0 & 0.9 & $1.2 \times 10^{27}$ \\
$I_{\text {fund }}\left(\mathrm{W} \mathrm{cm}{ }^{-2}\right)$ & 1.0 & 1.8 & 1.5 & 1.9 & $1.6 \times 10^{14}$ \\
Diameter $(\mathrm{mm})$ & 10 & 9 & 7.8 & 9 & 10 \\
Harmonic flux (arb. u.) & 0.7 & 1 & 1 & 1 & 0.8 \\
$\tau_{X U V}^{\text {meas }}(\mathrm{fs})$ & 41 & 31 & 23 & 28 & 36 \\
$\tau_{X U V}^{\text {sim }}(\mathrm{fs})$ & 39 & 17 & 15 & 24 & 30 \\
$b_{X U V}^{\text {meas }}\left(\mathrm{s}^{-2}\right)$ & -14 & -11 & -10 & 10 & $13 \times 10^{27}$ \\
$b_{X U V}^{\text {sim }}\left(\mathrm{s}^{-2}\right)$ & -17 & -33 & -7 & 7 & $18 \times 10^{27}$ \\
\hline \hline
\end{tabular}

makes the requirements on the MBES resolution less stringent.

Our numerical simulations of the sidebands are designed to mimic the experimental conditions as closely as possible. In particular, we use as input fields the fundamental and probe pulses retrieved from the SPIDER measurements. The output harmonic field is calculated by solving the Maxwell wave equation in the slowly varying envelope and paraxial approximations [22], using dipole moments and ionization rates obtained from integration of the time-dependent a)

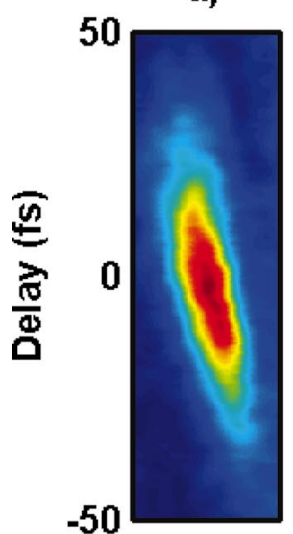

b)

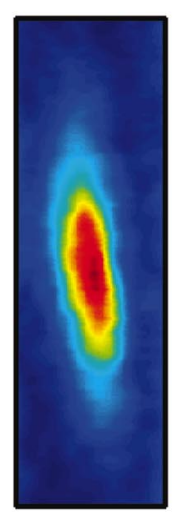

c)
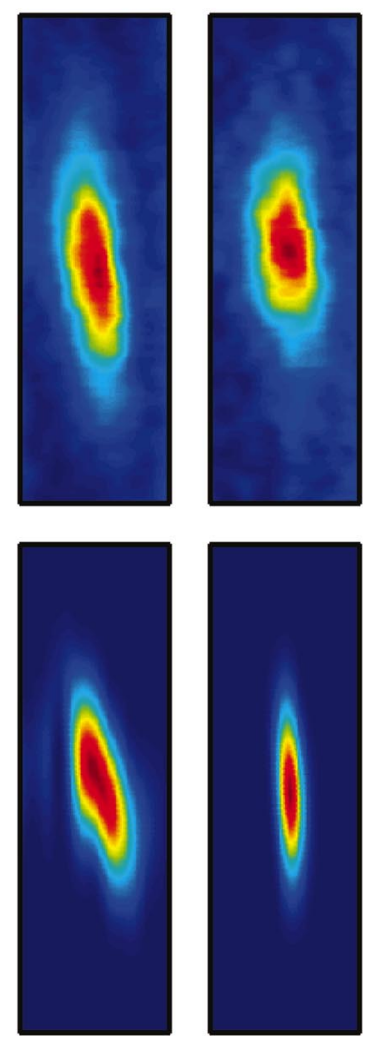

d)
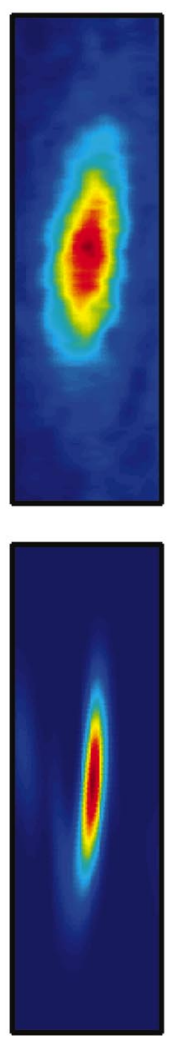

e)
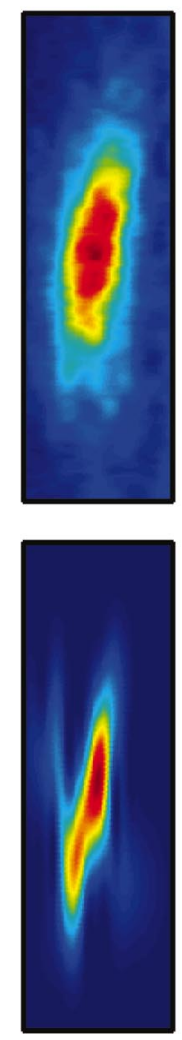

FIG. 3. (Color) Measured (top panel) and simulated (bottom panel) photoelectron signal corresponding to sideband 18 for negative (a), (b), zero (c), and positive (d), (e) fundamental chirp rates (see Table I for the chirp rate values). 
Schrödinger equation in the single active electron approximation [23]. The XUV field is then propagated over a $35 \mathrm{~cm}$ distance, where it is filtered through an aperture (as in the experimental setup) before being focused again into the interaction region. The sideband spectrum is calculated from

$$
S(\omega, \Delta t)=\left|\int_{-\infty}^{\infty} d t e^{i \omega t} E_{X U V}(t) E_{I R}(t-\Delta t)\right|^{2},
$$

where $E_{I R}, E_{X U V}$ are the electric fields of the IR and XUV pulses, $\Delta t$ is the delay between them, and $\omega$ is the laser frequency. This equation uses the framework of second-order perturbation theory, which is valid for the low probe intensities used in this experiment. The spectrum of a given sideband is obtained by adding two contributions, one from absorption of the $(q-1)$ th harmonic and an IR photon, and one from absorption of the $(q+1)$ th harmonic and emission of an IR photon.

Figure 2 shows a typical experimental result obtained using a fundamental field with a significant negative chirp [case (a) in Table I]. The photoelectron signals produced at the harmonic frequencies are shown by the saturated vertical stripes. The sidebands are visible between the harmonics. Their negative slope indicates that the harmonics are negatively chirped. In general, we find that the slope of the sidebands does not vary rapidly with order. We regard the chirp deduced from a given sideband $q$ as the average chirp of harmonics $q-1$ and $q+1$.

Figure 3 presents a comparison of experimental and theoretical data for one sideband, obtained using fundamental pulses with different spectral phases. In the experiment these phases are varied by introducing glass plates in either the probe beam or the beam generating the harmonics, while at the same time changing the distance between the gratings in the compressor to keep the probe pulse transform limited. The group delay dispersion (GDD) is determined from SPIDER measurements accounting for the effect of the entrance windows. An increase of the magnitude of the GDD leads to an increase in the fundamental pulse duration (see Table I). The chirp rate first increases in absolute value, reaches the maximum possible for the available bandwidth, corresponding to cases (b) and (e), and then decreases [case (a)]. The resulting decrease in peak intensity, which leads to a drop in harmonic yield, is partially compensated for by increasing the aperture before the focusing mirror (see Fig. 1). In both the experiment and simulation, varying the spectral phase of the fundamental pulse has a dramatic effect on the tilt of the sidebands. For all the cases shown in the figure the tilt of the sideband has the same sign as that of the chirp on the fundamental pulse. We also note that when the fundamental pulse is transform limited [case (c)], the harmonics exhibit a negative chirp.

The measured and simulated durations and chirp rates of the XUV pulses are presented in Table I, lines seven through ten. Experimentally, $\tau_{X U V}^{\text {meas }}$ is obtained from the duration of the sideband $\tau_{S B}$ according to $\tau_{X U V}^{\text {meas }}=\sqrt{\tau_{S B}^{2}-\tau_{I R}^{2}-\tau_{\text {geo }}^{2}}$, where the infrared probe pulse duration $\tau_{I R}$ is obtained from SPIDER measurements, and $\tau_{g e o}$ is a geometrical factor accounting for the slightly noncollinear geometry of our ex-

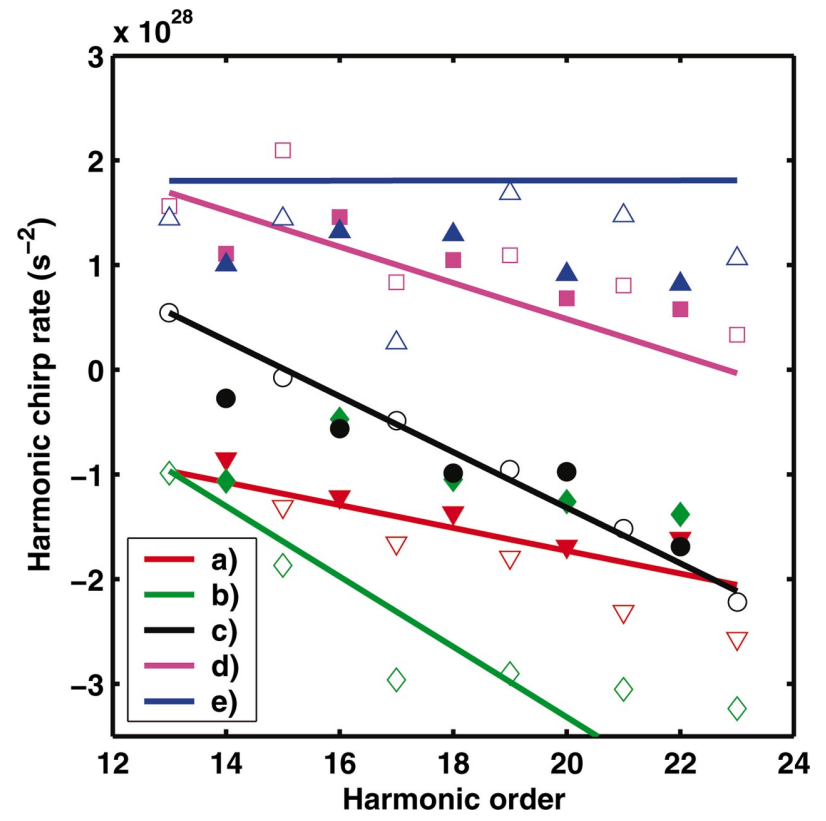

FIG. 4. (Color) Measured (closed symbols) and simulated (open symbols) chirp rates as a function of harmonic order for different fundamental spectral phases in Table I: (a) red, (b) green, (c), black, (d) purple, and (e) blue. The measured chirp rates are plotted as a function of the sideband order $q$, and include contribution from harmonics $q-1$ and $q+1$. The theoretical chirp rates are shown as functions of harmonic order, since these can easily be extracted from the simulations. The lines are explained in the text.

periment (estimated to be $18 \mathrm{fs}$ ). The chirp rate is then proportional to the sideband chirp rate $b_{\mathrm{SB}}: b_{X U V}^{\text {meas }}=b_{S B}\left[1+\left(\tau_{I R}^{2}\right.\right.$ $\left.+\tau_{\text {geo }}^{2}\right) / \tau_{X U V}^{2}$. The XUV pulse durations vary between 0.5 and 0.7 times the fundamental pulse duration, which is consistent with the assumption that ionization is low. The XUV chirp rates are approximately a factor of 10 larger than the fundamental chirp rates. In general the agreement between experiment and theory in Table I is good. The calculated pulse durations are in all cases somewhat smaller than the experimental ones.

In order to gain more insight into the origin of the harmonic chirp rates, we study the order dependence of the chirp rates for the fundamental pulses shown in Table I. Figure 4 shows a comparison between experimental and theoretical results for the different cases. The agreement between the experiment and theory is very good for the cases (a), (c), (d), and (e). In the case of the large negative chirp on the fundamental (b), we have not been able to obtain good agreement between experiment and theory. This is likely because we have been unable to accurately determine the fundamental pulse width and intensity in this case.

The results presented in Figure 4 can be interpreted by noting that the harmonic chirp rates in general have contributions from three different sources. First there is the temporal variation of the dipole phase $\phi_{q}(t)=-\alpha_{q} I(t)$, related to the electron excursion time for a given quantum orbit. Second, there are contributions from macroscopic timedependent processes, most importantly ionization. In our experimental conditions, we estimate that the ionization is less than $5 \%$ in all the cases. Along with allowing us to neglect 
the ionization contribution to the chirp, this means that the harmonics are generated close to the peak of the driving pulse. In this case, the dipole chirp contribution is linear and can be expressed as $b_{q}=-8 \ln (2) \alpha_{q} I_{\text {fund }} / \tau_{\text {fund }}^{2}$ for a Gaussian pulse. Third, there is a contribution from the spectral phase of the fundamental, which in these cases is close to a linear chirp. In the single atom response, the fundamental chirp rate $b_{\text {fund }}$ is simply transferred to the $q$ th harmonic as $q b_{\text {fund }}$, as expected in an adiabatic approximation. The propagation calculations within the slowly varying envelope approximation obviously do not change this result.

These considerations allow us to extract $\alpha_{q}$ from the results obtained using a transform-limited pulse [case (c), black symbols in Fig. 4]. We find that the magnitude of $\alpha_{q}$ increases with harmonic order, which is characteristic of the shortest quantum orbit [12]. It dominates due to the spatial selection achieved by the $1 \mathrm{~mm}$ hole through which the harmonic field propagates $[13,14]$. The second quantum path would lead to much larger (negative) chirps, decreasing in magnitude for increasing orders. The black line in Fig. 4 is a linear fit to the simulated chirp rates in case (c). We can, in turn, use these values for $\alpha_{q}$ to calculate the expected chirp rates in the other four cases with the simple formula: $q b_{\text {fund }}-8 \ln (2) \alpha_{q} I_{\text {fund }} / \tau_{\text {fund }}^{2}$, where we use $I_{\text {fund }}$ and $\tau_{\text {fund }}$ from Table I. The results are shown as the red, green, purple, and blue lines for cases (a), (b), (d), and (e), respectively. The fact that the expected values nicely overlap the experimental and simulated results indicates the robustness of the transfer of the fundamental chirp rate to the harmonics. Even in case (b) the simple chirp rate estimate agrees well with the simulation, though not the experiment. We note that the scaling of the dipole chirp with $I_{\text {fund }} / \tau_{\text {fund }}^{2}$ greatly reduces its contribution for the longer fundamental pulse [(case (a)]. In case (e), the two contributions to the chirp rate approximately cancel to give a chirp rate that is close to constant.

The results presented above demonstrate that we can modify the coherence properties of the harmonics through the spectral phase and the duration of the driving laser pulse. We expect these results to be valid also for higher-order harmonics. This method allows us to exert control over the temporal properties of an attosecond pulse train. In this experiment, we have found that to a good approximation the chirp rate varies with order as $b_{q}=\beta+q \gamma$. The attosecond train produced by these harmonics can be expressed as $E(t)$ $\propto \sum \exp \left(i q \omega t+i \beta t^{2} / 2+i q \gamma t^{2} / 2\right)$. The last term implies that the periodicity of the train $\left(T_{0}=\pi / \omega\right)$, will become time dependent according to $T(t)=T_{0}-\pi \gamma t / \omega^{2}$. For a negative $\gamma$, the spacing between the pulses will increase throughout the pulse. In the five cases studied here, we find $\Delta T=T(t)-T_{0}$ $=6 \times 10^{-4} t, 1.9 \times 10^{-3} t, 1.5 \times 10^{-3} t, 1 \times 10^{-3} t$, and 0 from (a) to (e). In our conditions, the effect of this temporal variation of the period remains small, about 60 as in case (c) over the total pulse. These effects have a negligible influence on the pulse duration of the attosecond pulses.

In conclusion, we have measured harmonic chirp rates as a function of order. This allows us to obtain information on how the single atom attosecond dynamics evolves during the driving laser pulse, and is thus complementary to the timeaveraged method used to characterize attosecond pulse trains $[4,6]$. The linear variation of the (negative) chirp rate with order that we have measured means that attosecond pulse trains synthesized from these harmonics have a pulse spacing that increases linearly with time. In the semiclassical model, which describes our results very well, this implies an increase in the harmonic emission time relative to the laser period for lower laser intensities, i.e., at the leading and trailing edges of the driving pulses [6]. We have also demonstrated a method for manipulating this emission time by using chirped fundamental pulses. In particular, we demonstrated that we can make a "perfect" train (i.e., equidistant as pulses) by canceling the dipole phase variation with a small positive chirp on the fundamental.

We acknowledge the support of the European Community's Human Potential Programme under Contract No. HPRNCT-2000-00133 (ATTO), the Knut and Alice Wallenberg Foundation, and the Swedish Science Council. K. S. acknowledges the support from the National Science Foundation through Grant No. PHY-9733890. K. V. is on leave from the Department of Optics and Quantum Electronics, University of Szeged, Szeged, Hungary. We also acknowledge the help from Fredrik Österberg at Rejlers for the support with the 3D figure. W. K., J. B., and U. K. were supported by the Swiss National Science Foundation and by Bundesamt für Bildung und Wissenschaft, Schweiz, Project BBW No. 03.0010 .
[1] M. Ferray et al., J. Phys. B 21, L31 (1988).

[2] L. Nugent-Glandorf et al., Phys. Rev. Lett. 87, 193002 (2001).

[3] S. Zamith et al., J. Chem. Phys. 119, 3763 (2003).

[4] P. M. Paul et al., Science 292, 1689 (2001).

[5] M. Hentschel et al., Nature (London) 414, 511 (2001).

[6] Y. Mairesse et al., Science 302, 1540 (2003).

[7] M. Drescher et al., Nature (London) 419, 803 (2002).

[8] K. J. Schafer et al., Phys. Rev. Lett. 92, 023003 (2004).

[9] J. Norin et al., Phys. Rev. Lett. 88, 193901 (2002).

[10] T. Sekikawa et al., Phys. Rev. Lett. 88, 193902 (2002).

[11] T. Sekikawa, T. Kanai, and S. Watanabe, Phys. Rev. Lett. 91, 103902 (2003).

[12] M. Lewenstein et al., Phys. Rev. A 52, 4747 (1995).
[13] M. Bellini et al., Phys. Rev. Lett. 81, 297 (1998).

[14] P. Salières et al., Science 292, 902 (2001).

[15] M. B. Gaarde and K. J. Schafer, Phys. Rev. A 65, 031406 (2002)

[16] Z. Chang et al., Phys. Rev. A 58, R30 (1998).

[17] R. Trebino et al., Rev. Sci. Instrum. 68, 3277 (1997).

[18] M. Nisoli et al., Opt. Lett. 22, 522 (1997).

[19] C. Iaconis and I. A. Walmsley, Opt. Lett. 23, 792 (1998).

[20] C. Iaconis et al., IEEE J. Quantum Electron. 35, 501 (1999).

[21] W. Kornelis et al., Opt. Lett. 28, 281 (2003).

[22] A. L'Huillier et al., Phys. Rev. A 46, 2778 (1992).

[23] K. J. Schafer and K. C. Kulander, Phys. Rev. Lett. 78, 638 (1997). 